\title{
Efficient extrapolation methods for electro- and magneto- quasistatic field simulations
}

\author{
M. Clemens, M. Wilke, and T. Weiland
}

Technische Universität Darmstadt, Dept. of Electrical Engineering and Information Technology, Computational Electromagnetics Laboratory (TEMF), Schloßgartenstr. 8, 64289 Darmstadt, Germany

\begin{abstract}
In magneto- and electroquasi-static time domain simulations with implicit time stepping schemes the iterative solvers applied to the large sparse (non-)linear systems of equations are observed to converge faster if more accurate start solutions are available. Different extrapolation techniques for such new time step solutions are compared in combination with the preconditioned conjugate gradient algorithm. Simple extrapolation schemes based on Taylor series expansion are used as well as schemes derived especially for multi-stage implicit Runge-Kutta time stepping methods. With several initial guesses available, a new subspace projection extrapolation technique is proven to produce an optimal initial value vector. Numerical tests show the resulting improvements in terms of computational efficiency for several test problems.
\end{abstract}

In quasistatischen elektromagnetischen Zeitbereichsimulationen mit impliziten Zeitschrittverfahren zeigt sich, dass die iterativen Lösungsverfahren für die großen dünnbesetzten (nicht-)linearen Gleichungssysteme schneller konvergieren, wenn genauere Startlösungen vorgegeben werden. Verschiedene Extrapolationstechniken werden für jeweils neue Zeitschrittlösungen in Verbindung mit dem präkonditionierten Konjugierte Gradientenverfahren vorgestellt. Einfache Extrapolationsverfahren basierend auf Taylorreihenentwicklungen werden ebenso benutzt wie speziell für mehrstufige implizite Runge-Kutta-Verfahren entwickelte Verfahren. Sind verschiedene Startlösungen verfügbar, so erlaubt ein neues Unterraum-Projektion-Extrapolationsverfahren die Konstruktion eines optimalen neuen Startvektors. Numerische Tests zeigen die aus diesen Verfahren resultierenden Verbesserungen der numerischen Effizienz.

Correspondence to: $\mathrm{M}$. Clemens

(clemens/wilke/weiland@temf.tu-darmstadt.de)

\section{Introduction}

Spatial discretizations of transient quasistatic electromagnetic field formulations with e.g. the Finite Element Method (FEM) or the Finite Integration Technique (FIT), commonly result either in large stiff ordinary differential or index 1 differential-algebraic systems of equations of the form

$\mathbf{D} \frac{d}{d t} \mathbf{x}(t)+\mathbf{K}[\mathbf{x}(t)] \mathbf{x}(t)=\mathbf{b}(t)$,

with the $N$-component vector $\mathbf{x}(t) \in \mathbb{R}^{N}$ whose evolution in time has to be considered over a given time interval. For magneto-quasistatic formulations see e.g. Kameari (1990) or Clemens and Weiland (1999) and for electro-quasistatic systems Preis et al. (2002) or Clemens et al. (2002a).

The solution of (1) requires to use suitable implicit time integration schemes, e.g. one-step schemes such as the simple $\theta$-methods, the multi-stage embedded (singly diagonal) implicit Runge-Kutta ((SD)IRK) methods in Cameron (1999) and linear-implicit time integration schemes of RosenbrockWanner-type in Hairer and Wanner (1996) or Lang (2001), which were just recently introduced to quasistatic electromagnetic field simulations in Clemens et al. (2002c), or the multi-step backward differentiation schemes described in Hairer and Wanner (1996). In these methods in each time step one or several large (non)linear algebraic systems of equations have to be solved. For the iterative solution methods of these systems extrapolation schemes can provide suitable initial start values. Such first approximations of the solutions at the respective new time steps should be computationally cheap to attain and allow to effectively reduce the number of subsequent iteration steps of the preconditioned conjugate gradient solvers. This was shown e.g. in Clemens et al. (2002d), where several extrapolation techniques are compared to produce an initial vector $\mathbf{x}_{0}^{(n+1)}$ for the iterative solution $\mathbf{x}^{(n+1)} \approx \mathbf{x}\left(t^{n+1}\right)$ of the algebraic system at time $t^{(n+1)}$

$\mathbf{M} \mathbf{x}^{(n+1)}=\mathbf{b}^{(n+1)}$, 
with $\mathbf{M}=[\alpha \mathbf{D}+\mathbf{K}]$, where $\alpha$ is a scalar parameter depending on the chosen time step length $\Delta t$.

This paper compares several techniques to improve the numerical performance of these algorithms, i.e., to reduce the required number of iterations in the solution methods for the linear algebraic systems of equations as e.g. in the preconditioned conjugate gradient (PCG) method. These scheme are here mainly used in the context of FIT-based magnetic field formulations, but extend also to other simulations where implicit time integration schemes are used with iterative solution methods. No benefits will arise from the presented methods if direct Gauss elimination schemes are applied which do not require start values.

\section{Simple extrapolation techniques in implicit time inte- gration schemes}

For each new time step solution $\mathbf{x}^{(n+1)} \approx \mathbf{x}\left(t^{(n+1)}\right)$ of the linear(-ized) system $\mathbf{M} \mathbf{x}^{(n+1)}=\mathbf{b}^{(n+1)}$ suitable initial solutions $\mathbf{x}_{0}^{(n+1)}$ for the iterative solution to start on have to be provided. The iterative conjugate gradient solution process is based on a Lanczos orthogonalization where a spectral approximation of the system matrix with tridiagonal matrices is performed as shown in van der Sluis and van der Vorst (1986). Thus the complete reduction of the spectral error components in the approximate solution should not benefit from an available good start solution $\mathbf{x}_{0}$. However, since the PCG process will be terminated when a user-specified finite accuracy is attained, small error components of the start vectors may not require additional iterations. As simple strategies for the choice of the start vectors we consider the following options that arise with Taylor series expansion extrapolations of zero-th order

$\mathbf{x}_{0}^{(n+1)}:=0$,

with first order

$\mathbf{x}_{0}^{(n+1)}:=\mathbf{x}^{(n)}$

or with second order

$\mathbf{x}_{0}^{(n+1)}:=\mathbf{x}^{(n)}+\Delta t^{(n+1)} \frac{d}{d t} \mathbf{x}^{(n)}$.

Starting the iterations using a homogeneous start vector $\mathbf{x}_{0}^{(n+1)}:=0$ may be expected to require the most iterative work, although it will best allow the conjugate gradient scheme to maintain its weak divergence property for nongauged, i.e., singular, magneto-quasistatic vector potential formulations (Cp. Clemens and Weiland (1999)). A more commonly used choice of an initial vector for the iterations at time $t^{n+1}$ consists in the solution of the previous time step $\mathbf{x}^{(n)}$ under the assumption that provided a short enough time step $\Delta t=t^{n+1}-t^{n}$ the solutions will not differ too much, i.e., using a first order Taylor approximation. An extension of equation (4) is to choose a second order Taylor expansion which involves the evaluation of the vector $\frac{d}{d t} \mathbf{x}^{(n)}$. It should be noted that in magnetodynamic simulations the calculation of the eddy current vector $j_{e c}^{(n+1)}=-\mathbf{M} k a p \frac{d}{d t} \mathbf{x}^{(n+1)}$ requires to evaluate these time derivative vectors anyway. If the chosen implicit time integration scheme does not provide an approximation also for $\frac{d}{d t} \mathbf{x}^{(n+1)}$ it can be calculated separately, for instance by using a second order one-step update scheme

$\frac{d}{d t} \mathbf{x}^{(n+1)} \approx \frac{2}{\Delta t}\left(\mathbf{x}^{(n+1)}-\mathbf{x}^{(n)}\right)-\frac{d}{d t} \mathbf{x}^{(n)}$

or using a BDF-formula, as e.g. the multi-step BDF2-approximation

$\frac{d}{d t} \mathbf{x}^{(n+1)} \approx \frac{1}{\Delta t}\left(\frac{3}{2} \mathbf{x}^{(n+1)}-2 \mathbf{x}^{(n)}+\frac{1}{2} \mathbf{x}^{(n-1)}\right)$.

These simple and cost effective to implement start value selection strategies based on the Taylor series expansion can be applied to any suitable implicit time integration scheme.

A similarly simple and seemingly straightforward approach for an extrapolation strategy arises with

$\mathbf{x}_{0}^{(n+1)}:=\tilde{\mathbf{M}}^{-1} \mathbf{b}^{(n+1)}$

which involves the use of an easily invertible preconditioning matrix $\widetilde{\mathbf{M}}$ approximating the system matrix M. A simple choice is e.g. to take the SSOR-preconditioning matrix $\widetilde{\mathbf{M}}_{\mathrm{SSOR}}=(\mathbf{D}-\mathbf{L}) \mathbf{D}^{-\mathbf{1}}(\mathbf{D}-\mathbf{U})$, with $\mathbf{M}=\mathbf{D}-\mathbf{L}-\mathbf{U}$.

Since the first step within a PCG method actually consists in the application of (8), no considerable improvements of the process convergence are to be expected from this strategy. Numerical tests in Clemens et al. (2002d) confirm this. These simulation results also indicate that using only the start strategy in (4) may be advantageous for constant or nearly constant current excitations whereas the second order Taylor extrapolation in (5) was tested with better results for current excitations with strong variations in the considered time interval. Thus it is not initially clear which strategy to follow.

\section{Extrapolation techniques for multi-stage SDiRK methods}

In the past years, higher order singly diagonal implicit Runge-Kutta (SDiRK) methods were established to be suitable methods for transient magnetic field simulations in Nicolet and Delincé (1996). The increased interest in this family of time integration methods for transient magnetic fields arises from the fact that these one-step methods provide stiffly accurate schemes of almost arbitrarily high order well suited for numerical time integration of differential-algebraic systems of equations of Index 1 of magnetodynamic formulations. An $s$-stage SDiRK scheme requires to solve $s$ (non)linear algebraic systems of equations to yield the stage variables $\mathbf{Y}_{i}$, i.e., intermediate solution vectors, to be evaluated at times $t_{i}=t^{(n)}+c_{i} \Delta t$ with

$$
\mathbf{Y}_{i}^{(n+1)}=\mathbf{x}^{(n)}+\Delta t \sum_{j=1}^{i} a_{i j} \mathbf{Y}_{i}^{\prime}, \quad i=1, \ldots, s,
$$


involving the previous time step solution $\mathbf{x}^{(n)}$, the stage derivatives $\mathbf{Y}_{i}^{\prime}$, the SDiRK-coefficient matrix $\mathbf{A}=$ $\left\{a_{i j}\right\}_{1 \leq i, j \leq s},\left(a_{i j}=0\right.$ for all $\left.i<j\right)$. The new time step solution of the SDiRK-scheme is given by

$\mathbf{x}^{(n+1)}=\mathbf{x}^{(n)}+\Delta t \sum_{j=1}^{s} b_{j} \mathbf{Y}_{i}^{\prime}$.

The method coefficient vectors $\mathbf{b}=\left\{b_{j}\right\}_{1 \leq j \leq s}, \mathbf{c}=$ $\left\{c_{j}\right\}_{1 \leq j \leq s}$, and the method matrix A completely specify the chosen SDiRK method. The internal multi-stage construction principle in some cases also allows to construct embedded schemes corresponding to a second coefficient vector $\hat{\mathbf{b}}$ to provide an additional solution of a lower order. In Cameron et al. (1998) a stiffly accurate, L-stable 4-stage SDiRK3(2) scheme of third (embedded scheme: second) order was proposed and its use for the purposes of an errorcontrolled variable step length time integration of the discrete magnetodynamic systems based on Finite-Element and Finite Integration method formulations was further refined in Wang et al. (2001) and Clemens et al. (2002b).

For the SDiRK schemes we consider the following strategies to choose a suitable start value. The most simple extrapolation formulation is given with a first order Taylor series expansion extrapolation

$\mathbf{Y}_{i, 0}^{(n+1)}:=\mathbf{x}^{(n)}$,

where the last time step solution $\mathbf{x}^{(n)}$ is taken as start value for each stage variable vector to be calculated for the next time step.

It is, however, also possible to use the intermediate solutions of the internal SDiRK-stages to construct extrapolated start vectors $\mathbf{Y}_{i, 0}^{(n+1)}$ for the solution at the new time step $t^{(n+1)}$. An extensive mathematical treatment of these techniques is found in Cameron (1999), where a stage extension extrapolation method is described with

$$
\mathbf{Y}_{i, 0}^{(n+1)}:=\left\{\begin{array}{l}
\mathbf{x}^{(n)}: \quad i=1, \\
\mathbf{Y}_{l}^{(n+1)}: \text { for } c_{l}=\max _{1 \leq k<i}\left\{c_{k} \mid c_{k} \leq c_{i}\right\} \\
\frac{c_{i}-c_{j}}{c_{k}-c_{j}} \mathbf{Y}_{j}^{(n+1)}+\frac{c_{k}-c_{i}}{c_{k}-c_{j}} \mathbf{Y}_{k}^{(n+1)}: \\
\quad c_{i} \text { for } c_{l} \leq c_{j} \leq c_{i} \leq c_{k} \leq c_{m} \\
\quad \text { for all } l \leq j, k<i ; k \leq m
\end{array},\right.
$$

where a start solution for the corresponding stage value vector is constructed using already available stage values of the current time step $\Delta t^{(n+1)}=t^{(n+1)}-t^{(n)}$. Not for every SDiRK method the coefficients $c_{i}$ of the intermediate times $t^{(n)}+c_{i} \Delta t^{(n)}$ increase monotonically in size such that $c_{i} \leq c_{j}, i>j$, holds. Thus, either an already available previous stage value is used as start solution or an interpolation is performed using the nearest in time stage values $\mathbf{Y}_{j}$ and $\mathbf{Y}_{k}$ with $c_{j} \leq c_{i} \leq c_{k}$ following a method described in Cameron (1999). Note, that certain methods allow to consider two stage variable vectors $\mathbf{Y}_{i}, \mathbf{Y}_{j}, i \neq j$, at the same time point in the integration interval, i.e., $c_{i}=c_{j}$.

A variant of this approach is the continuous extension extrapolation scheme. The solution vectors $\overline{\mathbf{x}}$ at times $t^{\star}=$ $t^{(n)}+\Delta t^{(n)}+c_{i} \Delta t^{(n+1)}:=t^{(n)}+\sigma_{i} \Delta t^{(n)}$, can be extrapolated from the stage derivatives of the last time step by

$\mathbf{Y}_{i, 0}^{(n+1)}:=\overline{\mathbf{x}}\left(t^{\star}\right)=\mathbf{x}^{(n)}+\sigma_{i} \Delta t \sum_{j=1}^{s} \bar{b}_{j}\left(\sigma_{i}\right) \mathbf{Y}_{i}^{\prime}$,

which involves a coefficient vector $\overline{\mathbf{b}}=\left\{\bar{b}_{j}\right\}_{1 \leq j \leq s}$, to achieve the extrapolated stage variable $\mathbf{Y}_{i, 0}^{(n+1)}$ of the new time step.

For the SDiRK3(2) scheme the following extrapolator coefficient set is available from Cameron (1999) with

$$
\begin{aligned}
& \overline{\mathbf{b}}^{T}(\sigma):=\frac{1}{30}\left[\begin{array}{lll}
1 & \sigma & \sigma^{2}
\end{array}\right] \\
& {\left[\begin{array}{cccc}
22 \sqrt{2}+15 & 236-159 \sqrt{2} & 1-7 \sqrt{2} & 135 \sqrt{2}-222 \\
-14 \sqrt{2}-15 & 135 \sqrt{2}-262 & 13+14 \sqrt{2} & 264-135 \sqrt{2} \\
2 \sqrt{2}+5 & 16+20 \sqrt{2} & -9-7 \sqrt{2} & -12-15 \sqrt{2}
\end{array}\right] .}
\end{aligned}
$$

Note, that one-stage SDiRK schemes are closely related to the standard one-step $\theta$-methods described e.g. in Hairer and Wanner (1996). For these time integration methods the strategies in Eqs. (11) and (12) will coincide with method (4) and strategy (13) is identical to method (5). Another specific advantage of stiffly accurate SDiRK arises from the fact that in these methods we have the last intermediate time step coefficient $c_{s}=1$, such that $\mathbf{Y}_{s}^{\prime}=\mathbf{Y}^{\prime}\left(t^{(n+1)}\right) \approx \frac{d}{d t} \mathbf{x}\left(t^{(n+1)}\right)$ holds. Accordingly, no additional evaluations for the time derivative vectors $\frac{d}{d t} \mathbf{x}\left(t^{(n+1)}\right.$ have to be performed.

\section{Hybrid extrapolation techniques}

All the start vectors $\mathbf{x}_{0, i}, i=1, \ldots, m$, derived from the extrapolation techniques described above for the iterative solution of (2) are computationally cheap to attain, but numerical test in Clemens et al. (2002d) indicate that just one extrapolation method used for the generation of start values may not be suitable for all kind of transient problems.

In order to solve this problem, in Clemens et al. (2002d) already a minimal residual norm selection criterion has been proposed for a set of start values $\mathbf{x}_{0, i}, i=1, \ldots, m$, constructed with different extrapolation methods. In this minimal norm hybrid extrapolation scheme first the norms of residual vectors for the different start solutions $\mathbf{x}_{0, i}$ are evaluated with low additional computational costs. The vector $\mathbf{x}_{0, j}$ corresponding to the smallest residual norm is chosen as a start vector:

$$
\begin{aligned}
r_{i} & :=\left\|\mathbf{M x}_{0, i}-\mathbf{b}^{(n+1)}\right\|_{2}, \quad i=1, \ldots, m, \\
\mathbf{x}_{0}^{(n+1)} & :=\mathbf{x}_{0, j}, \text { for } j: \quad r_{j}=\min _{i=1, \ldots, m}\left\{r_{i}\right\} .
\end{aligned}
$$

The numerical tests show that this approach, while rather simple, is surprisingly effective for a robust reduction of the required computational work independent of the current excitation form.

A more refined technique for the construction of a start solution vector for system (2) is available with the novel subspace projection extrapolation (SPE) technique (SPE). 


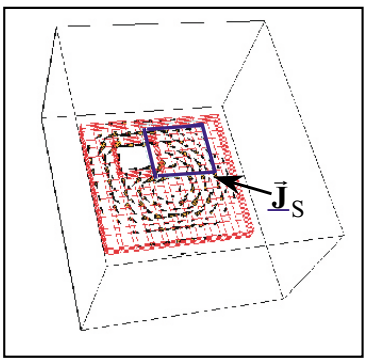

(1)

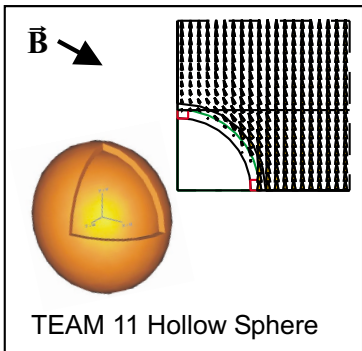

(2)

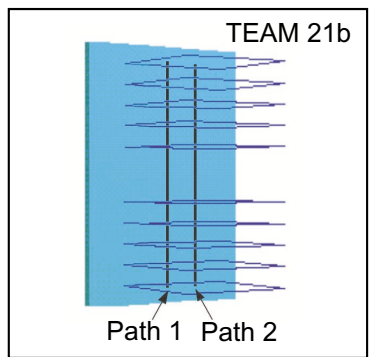

(3)

Fig. 1. Three eddy current test problems: 1) A copper plate with a hole: Time harmonic eddy currents in a conductive plate. 2) The TEAM 11 problem: A conductive sphere in an abruptly started homogeneous vertical magnetic field 3) The TEAM 21b problem: Time harmonic eddy currents in a ferromagnetic nonlinear iron plate.

Table 1. Extrapolation strategies for a fixed time stepping BDF1-scheme. The numbers denote the total number of matrix-vectormultiplications during the complete time integration process.

\begin{tabular}{lccc}
\hline Problem & No. 1 & No. 2 & No. 3 \\
\hline (a) Taylor 1st order $\mathbf{x}_{0}^{(n+1)}:=\mathbf{x}^{(n)}$ & 3424 & 6663 & - \\
(b) Taylor 2nd order $\mathbf{x}_{0}^{(n+1)}:=\mathbf{x}^{(n)}+\Delta t \dot{\mathbf{x}}^{(n)}$ & 2554 & 16730 & - \\
(c) Minimal norm hybrid method for (a), (b) & 2714 & 6703 & 74392 \\
(d) SPE scheme using (a), (b) $(m=2)$ & 923 & 2889 & 41182 \\
\hline
\end{tabular}

For this we require that the system matrix $\mathbf{M}$ in (2) has to be symmetric and positive (semi-)definite, which is commonly the case in discrete electro- and magneto-quasistatic field formulations. Here the initial vectors $\mathbf{x}_{0, i}, i=1, \ldots, m$, are orthonormalized using a Modified Gram Schmitt procedure (see e.g. Meister (1999)) to yield the vectors $\mathbf{v}_{0, i}, i=$ $1, \ldots, \tilde{m}$, which form a orthonormal basis to a vector subspace $V_{\tilde{m}}=\operatorname{span}\left\{\mathbf{v}_{j}\right\}=\operatorname{span}\left\{\mathbf{x}_{0, i}\right\} \subseteq \mathbb{R}^{N}$ which is spanned already by all the different start vectors $\mathbf{x}_{0, i}$. Since the orthonormalization process will detect linear dependencies in the $m$ original vectors $\mathbf{x}_{0, i}$, the relation $\tilde{m} \leq m$ will hold.

With the definition of the orthonormal operator $\mathbf{V}:=$ $\left\{\mathbf{v}_{1}|\ldots| \mathbf{v}_{\tilde{m}}\right\} \in \mathbb{R}^{N \times \tilde{m}}$, we can restrict the system (2) onto $V_{\tilde{m}}$

$\left[\mathbf{V}^{T} \mathbf{M V}\right] \mathbf{z}=\mathbf{V}^{T} \mathbf{b}^{(n+1)}$.

to achieve an optimal approximative solution $\mathbf{x}_{0}^{(n+1)}:=\mathbf{V z}$ of (2) inside $V_{\widetilde{m}}$. Note, that the solution of (15) commonly involves a $\widetilde{m} \times \tilde{m}$ matrix with $\widetilde{m} \ll 100 \ll N$. Thus a direct Gauss elimination process can be adopted and the inverse $\left[\mathbf{V}^{T} \mathbf{M V}\right]^{-1}$ of the system matrix is available. With this the subspace projection extrapolation can be summarized as

$\mathbf{x}_{0}^{(n+1)}=\mathbf{V}\left[\mathbf{V}^{T} \mathbf{M V}\right]^{-1} \mathbf{V}^{T} \mathbf{b}^{(n+1)}$.

For the resultant initial vector $\mathbf{x}_{0}^{(n+1)}$ the Galerkin condition $\mathbf{V}^{T}\left[\mathbf{b}^{(n+1)}-\mathbf{M} \mathbf{x}_{0}^{(n+1)}\right]=0$ holds w.r.t. $V_{\widetilde{m}}$. Such a Galerkin condition is also essential for the construction of the iterative conjugate gradient solution process.

The subspace projection extrapolation scheme essentially yields an optimal start solution for the system (2) restricted to the linear subspace $V_{\widetilde{m}} \subset \mathbb{R}^{N}$ spanned by the different start vectors $\mathbf{x}_{0, i}$. In principle it may occur that there is no reduction of the iteration steps for the start values generated with this SPE method if the subsequently applied conjugate gradient process is considered as an exact method. Beneficial effects, however, will arise in approximative solution processes, where the iterations are terminated once a prescribed accuracy for the relative norms of the residual vectors $\left\|\mathbf{M} \mathbf{x}_{k}^{(n+1)}-\mathbf{b}^{(n+1)}\right\| /\left\|\mathbf{M} \mathbf{x}_{0}^{(n+1)}-\mathbf{b}^{(n+1)}\right\| \leq \varepsilon_{\mathrm{PCG}}$ is reached.

For many electro- or magneto-quasistatic problems described with (1) the matrix $\mathbf{M}=\mathbf{M}(\mathbf{x})$ will, however, depend on the solution vector. Such behavior arises with the simulation of nonlinear ferromagnetic materials in magnetoquasistatic problems or field-dependent conductivities in transient electro-quasistatic simulations. While the subspace projection extrapolation initially is derived as optimal start value generation scheme only for linear problems, in this nonlinear case it can be used to perform a few nonlinear fixpoint iteration steps $k=0,1, \ldots$,

$\mathbf{V}^{T} \mathbf{M}\left(\mathbf{x}_{0, k}\right) \mathbf{V} \mathbf{z}_{k+1}=\mathbf{V}^{T} \mathbf{b}^{(n+1)}, \mathbf{x}_{0, k+1}:=\mathbf{V} \mathbf{z}_{k+1}$,

where this iteration is started for $k=0$ with $\mathbf{x}_{0,0}:=$ $\mathbf{x}^{(n)}$. The iteration (17) corresponds to that of a SuccessiveApproximation method restricted to the subspace $V_{\widetilde{m}}$.

\section{Numerical results}

The extrapolation strategies presented above are compared for typical transient magnetic test configurations shown in Fig. 1 simulated with a modified vector potential formulation based on the Finite Integration Technique described 
Table 2. Extrapolation strategies for the adaptive SDIRK3(2)-scheme from Cameron (1999). The numbers denote the total number of matrix-vector-multiplications during the complete time integration process.

\begin{tabular}{lccc}
\hline Problem & No. 1 & No. 2 & No. 3 \\
\hline (a) Taylor 1st order $\mathbf{x}_{0}^{(n+1)}:=\mathbf{x}^{(n)}$ & 4636 & 12310 & 70647 \\
(b) Taylor 2nd order $\mathbf{x}_{0}^{(n+1)}:=\mathbf{x}^{(n)}+\Delta t \dot{\mathbf{x}}^{(n)}$ & 4473 & 10925 & 69946 \\
(c) Stage-extension extrapolation & 3877 & 7466 & 58799 \\
(d) Continuous extension extrapolation & 4145 & 10778 & 66660 \\
(e) Minimal norm hybrid method for (a),(c),(d) & 3777 & 6688 & 55198 \\
(f) SPE-scheme using (a),(c),(d) $(m=3)$ & 2743 & 6572 & 35743 \\
\hline
\end{tabular}

Table 3. Extrapolation strategies for the adaptive linear-implicit scheme RODAS3(2) described in Clemens et al. (2002c). The numbers denote the total number of matrix-vector-multiplications during the complete time integration process.

\begin{tabular}{lccc}
\hline Problem & No. 1 & No. 2 & No. 3 \\
\hline Zero start vector $\hat{\mathbf{v}}_{0}^{(n+1)}:=0$ & 8397 & 8135 & 87277 \\
SPE scheme using 3 last stage solutions $(m=3)$ & 4766 & 5537 & 45590 \\
\hline
\end{tabular}

in Clemens and Weiland (1999). Problem 1 is related to the TEAM 7 problem, a copper plate with a hole featuring a ramped $50 \mathrm{~Hz}$ sinusoidal current excitation simulated for 80 time steps. Problem 2 is a hollow conductive, nonferromagnetic sphere in an abrupt B-field (TEAM 11) integrated with 40 time steps. Problem 3 consists of the TEAM $21 \mathrm{~b}$ problem, a nonlinear $50 \mathrm{~Hz}$ time harmonic eddy current problem, simulated over 50 time steps (see also Clemens et al. (2002d)).

In Table 1 the effect of the new SPE method is displayed for the commonly used implicit BDF1 method and in Table 2 for a time-step adaptive 4-stage SDIRK3(2)-method introduced in Cameron (1999). The solution of the linear algebraic systems is performed with a SSOR-preconditioned CG method which terminates its iterations after having reached a relative accuracy $\varepsilon_{\mathrm{PCG}}=10^{-5}$. The comparison of the different start strategies is given in terms of the required total number of matrix vector multiplications ( $\mathrm{MxV}$-operations) for the time integration process. This number corresponds well to the required computational time.

The results for both the standard fixed time step BDF1scheme as well as the adaptive SDIRK3(2)-scheme show that the application of the SPE scheme speeds up the solution process by a factor $\geq 2$ for linear problems when compared to just using the 1st order Taylor expansion, i.e., starting the PCG-iterations with the old solution $\mathbf{x}_{0}^{(n+1)}:=\mathbf{x}^{(n)}$. The new SPE approach also outperforms all the other extrapolation methods presented above. The convergence of the nonlinear problem, in which 4 nonlinear SPE-cycles are performed before entering the nonlinear iteration scheme, is also considerably improved.

In Table 3 results for the linear-implicit time integration method RODAS3(2) described in Lang (2001) or Clemens et al. (2002c) are depicted. Its intermediate stage solutions, for which only linear systems have to be solved, no longer have interpolating character. Thus the extrapolation schemes available for SDIRK-methods are not directly applicable and zero start vectors are commonly used. The application of the new SPE scheme to its respective three last stage solutions $(m=3)$, however, allows to achieve a considerable speed-up of the PCG solver convergence.

\section{Conclusion}

For transient quasistatic electromagnetic field simulations with implicit time stepping schemes algorithmic improvements are presented. Different extrapolation techniques for new time step solutions are compared in combination with the preconditioned conjugate gradient algorithm. Simple extrapolation schemes are suitable for all standard implicit time integration schemes. Their ideas extend to specialized schemes for multi-stage implicit Runge-Kutta time stepping schemes. With the subspace projection extrapolation technique a new hybrid technique is proposed which allows to choose an optimal initial value independent of the problem dynamics at only moderate additional costs. Numerical tests illustrated the resulting improvements of the computational efficiency for several transient magnetodynamic problems.

\section{References}

Cameron, F.: Low-Order Runge-Kutta Methods for Differential Algebraic Equations, Ph.D. thesis, Tampere Univ. of Technology, 1999.

Cameron, F., Piché, R., and Forsman, K.: Variable step size time integration methods for transient eddy current problems, IEEE Trans. Magn., 34, 3319-3323, 1998. 
Clemens, M. and Weiland, T.: Transient eddy current calculation with the FI-method, IEEE Trans. Magn., 35, 1163-1166, 1999.

Clemens, M., Gersem, H. D., Koch, W., Wilke, M., and Weiland, T.: Transient simulation of nonlinear electro-quasistatic problems using the Finite Integration Technique, in Proceedings of the 10th International IGTE Symposium on Numerical Field Calculation in Electrical Engineering, 2002, Graz, Austria, pp. 510515, 2002a.

Clemens, M., Wilke, M., and Weiland, T.: 3D transient eddy current simulations using $\mathrm{FI}^{2} \mathrm{TD}$ with variable time step size selection schemes, IEEE Trans. Magn., 38, 605-608, 2002 b.

Clemens, M., Wilke, M., and Weiland, T.: Linear-implicit time integration schemes for error-controlled transient nonlinear magnetic field simulations, in Proc. CEFC,2002, Perugia, p. 332, to appear in IEEE Trans. Magn. (scheduled May 2003), 2002c.

Clemens, M., Wilke, M., and Weiland, T.: Extrapolation strategies in transient magnetic field simulations, in Proc. CEFC 2002, Perugia, p. 331, to appear in IEEE Trans. Magn. (scheduled May 2003), 2002d.

Hairer, E. and Wanner, G.: Solving Ordinary Differential Equations II, Stiff and Differential-Algebraic Problems, Springer, Wien,
New York, 1996.

Kameari, A.: Calculation of transient 3d eddy current using edge elements, IEEE Tr. Magn., 26, 466-469, 1990.

Lang, J.: Adaptive Multilevel Solution of Nonlinear Parabolic PDE Systems: Theory, Algorithm and Application, Springer-Verlag, Berlin, Heidelberg, New York, 2001.

Meister, A.: Numerik linearer Gleichungssysteme, Vieweg-Verlag, Braunschweig, Wiesbaden, 1999.

Nicolet, A. and Delincé, F.: Implicit Runge Kutta methods for transient magnetic field computation, IEEE Trans. Magn., 32, 14051408, 1996.

Preis, K., Bíró, O., Supancic, P., and Ticar, I.: FEM simulation of thermistors including dielectric effects, in Proceedings of the CEFC 2002, Perugia, Italy, 16.-19. June, edited by E. Cardelli, 2002.

van der Sluis, A. and van der Vorst, H. A.: The rate of convergence of conjugate gradients, Numer. Math., 48, 543-560, 1986.

Wang, H., Taylor, S., Simkin, J., Biddlecomb, C., and Trowbridge, B.: An adaptive-step time integration method applied to transient magnetic field problems, IEEE Trans. Magn., 37, 3478-3481, 2001. 